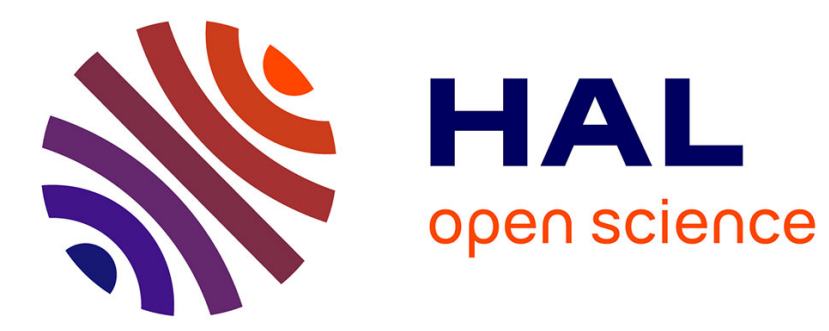

\title{
An Obstacle-Aware Clustering Protocol for Wireless Sensor Networks with Irregular Terrain
}

Riham Elhabyan, Wei Shi, Marc St-Hilaire

\section{To cite this version:}

Riham Elhabyan, Wei Shi, Marc St-Hilaire. An Obstacle-Aware Clustering Protocol for Wireless Sensor Networks with Irregular Terrain. International Conference on Wired/Wireless Internet Communication (WWIC), Jun 2018, Boston, MA, United States. pp.54-66, 10.1007/978-3-030-02931-9_5 . hal-02269728

\section{HAL Id: hal-02269728 \\ https://hal.inria.fr/hal-02269728}

Submitted on 23 Aug 2019

HAL is a multi-disciplinary open access archive for the deposit and dissemination of scientific research documents, whether they are published or not. The documents may come from teaching and research institutions in France or abroad, or from public or private research centers.
L'archive ouverte pluridisciplinaire HAL, est destinée au dépôt et à la diffusion de documents scientifiques de niveau recherche, publiés ou non, émanant des établissements d'enseignement et de recherche français ou étrangers, des laboratoires publics ou privés. 


\title{
An Obstacle-aware Clustering Protocol for Wireless Sensor Networks with Irregular Terrain
}

\author{
Riham Elhabyan, Wei Shi, and Marc St-Hilaire \\ School of Information Technology, Faculty of Engineering and Design \\ Carleton University, Ontario, Canada \\ \{riham.elhabyan, wei.shi, marc.sthilaire\}@carleton.ca
}

\begin{abstract}
Clustering in Wireless Sensor Networks (WSNs) is considered an efficient technique to optimize the energy consumption and increase the Packet Delivery Rate (PDR). Most of the proposed clustering protocols assume that there is a Line of Sight (LOS) between all the sensors. In real situations, there are obstacles which could interfere this LOS. Moreover, most of the available WSNs simulators assume the use of optimistic path loss models that neglect the effect of the obstacles on the PDR. In this paper, we adopt an obstacle-aware path loss model to reflect the effect of the obstacles on the communication between any the sensors. The Castalia simulator is then adapted to use this the proposed path loss model. Moreover, we propose an obstacle-aware clustering protocol, the NSGA-based, Non-LOS Cluster Head selection (NSGA-NLOS-CH) protocol, to solve the CHs selection problem in WSNs with an irregular field. Simulation results have shown that the effect of the obstacles on the PDR cannot be neglected. Moreover, NSGA-NLOS-CH outperforms other competent protocols in terms of the PDR while maintaining an acceptable energy consumption at the same time.
\end{abstract}

Keywords: Obstacle-aware $\cdot$ Clustering $\cdot$ WSNs

\section{Introduction}

Clustering in Wireless Sensor Networks (WSNs) is considered an efficient technique to optimize the energy consumption. Many clustering protocols have been proposed in the literature to optimize the energy consumption in Wireless Sensor Networks (WSNs). Most of these protocols assume that the sensors are deployed in a Two-Dimensional (2D) network field. However, there is an increasing number of WSNs applications in which the network field is often a Three-Dimensional (3D) rolling terrains, such as volcano monitoring and landslide detection. Although recent studies have proposed clustering protocols for 3D WSNs [7], these studies assume that the field is a 3D volume where sensors can be positioned freely within the whole 3D space. Compared to this free deployment in 3D space, in the case of deployment on rolling fields, sensors are deployed on the exposed surface and not freely within the 3D space. Therefore, these protocols cannot be applied to the rolling terrain either. Clustering protocols developed for $2 \mathrm{D}$ fields cannot be applied directly in such applications because the nature of the $3 \mathrm{D}$ rolling field may lead to the creation of obstacles in the network field. These 
obstacles have a substantial impact on the link quality between the communicating sensors as they cause an increased path loss. Therefore, determining the optimal set of CHs on the 3D rolling fields is a critical task.

Data delivery reliability is considered a key requirement in WSNs. In order to realize this requirement, clustering protocols should ensure high-quality links between the cluster members and their associated Cluster Heads (CHs). The Received Signal Strength Indicator (RSSI) is considered a prominent metric to assess the link quality between the transmitter and the receiver sensors. The RSSI calculation depends mainly on the adopted path loss model. Therefore, the performance of clustering protocols critically depends on the ability to accurately model the path loss of the communication signal between the transmitter and the receiver. A common limitation in most of the previously proposed clustering protocols is assuming the free space path loss model. The fundamental assumption behind this model is that the transmitter and the receiver sensors have a Line Of Sight (LOS) communication with no obstacles of any kind [8]. In real situations, there are almost always obstacles in the path between the transmitter and the receiver. Therefore, the free space path loss model is considered ideal and optimistic for predicting the path loss between any two sensors since it does not take the obstacles effect between the transmitter and the receiver into account [10]. Most of the available WSNs simulators assume the use of the free space path loss model[9]. The log-normal shadow fading model is proposed as an attempt to construct a more realistic path loss model by simulating the path loss around the sensors. Yet, this model does not account for the effect of the obstacles on the communication signal. Another significant limitation is that most of the existing clustering protocols assume the use of the first order radio model. However, this energy model is idealized and fundamentally flawed for modeling radio power consumption in sensor networks [5].

Castalia is a popular and very efficient WSN simulator that provides a welldesigned channel model [9] and adopts a realistic energy consumption model that is based on the characteristics of the Chipcon CC2420 radio transceiver data sheet. However, Castalia adopts the log-normal shadow fading model and hence shares the same drawback as most of the available WSNs simulators with regard to accurately modeling the path loss in case of No Line Of Sight (NLOS) communication, i.e. when there are obstacles between the transmitter and the receiver.

\subsection{Contributions}

As we discussed earlier, obstacles have a great impact on both the design of clustering protocols for WSNs and the simulation and evaluation of the performance of these protocols. Therefore, the purpose of this paper is twofold. First, to design, implement and test an obstacle-aware clustering protocol to solve the CHs selection problem in 3D rolling fields. Since the CHs selection problem has been proven to be a Non-deterministic Polynomial (NP)-hard problem with many conflicting objectives, a Pareto-based Evolutionary Algorithm (EA) is adopted to solve this problem. The proposed protocol takes into consideration the following key requirements: the network's energy efficiency, the network's data delivery 
reliability, and the protocol's scalability. Second, to test and investigate the performance of the proposed protocol against well-known clustering protocols, in the existence of obstacles and under a realistic energy consumption model. The main contributions of this paper are listed below:

- We adapt an obstacle-aware path loss model to evaluate the effect of the obstacles in the communication between any two sensor nodes. In order to achieve that, we implement a visibility function to find the obstacles in the path between any two sensors. This function is implemented based on the Bresenham's algorithm. Based on the adopted path loss model, a path loss map is derived. The Castalia simulator is then modified to use this map to calculate the path loss and the RSSI values between any two sensors in the network.

- We propose a new EA-based clustering protocol for 3D WSNs where the network field is a $3 \mathrm{D}$ rolling terrain.

- In order to realize the aforementioned contributions, the network field is modeled using the Digital Elevation Model (DEM) to account for different elevations, and hence obstacles, in the field.

The novelties of the proposed research work are as follows: a) To the best of our knowledge, the proposed clustering protocol is the first to consider and test the obstacles' effect on the communication between the sensors, b) Experimental validation are performed on real elevation data for 3D terrains.

\subsection{Paper Organization}

The remainder of this paper is organized as follows. Section 2 presents the related work on protocols designed for 3D WSNs. Section 3 presents the system model and assumptions. The design of the obstacle-aware clustering protocol and the problem formulation are provided in section 4 A detailed analysis of the simulations results is provided in Section 5. Finally, Section 6 concludes this paper and highlights future research directions.

\section{Related Works}

The Low Energy Adaptive Clustering Hierarchy for 3D WSNs (LEACH-3D) protocol [2] is a direct extension of the original LEACH protocol and is considered the first clustering protocol designed for 3D WSNs. The first order radio model, which is initially proposed by LEACH, is extended to work for 3D WSNs. Based on this extension, the authors prove that the effect of the 3D environment on clustering protocols cannot be neglected. LEACH-3D uses a variable number of $\mathrm{CHs}$ and different number of $\mathrm{CHs}$ could be elected each round.

A Fuzzy-based Clustering Scheme for 3D WSNs (FCM-3) is proposed in [7] to apply clustering protocols in 3D WSNs. The proposed protocol assumes the radio model which is proposed in [2]. The adopted fuzzy approach optimizes one objective function which is defined as minimizing the total energy consumption and is constructed by combining the distances between sensor nodes and their corresponding $\mathrm{CHs}$ and between the $\mathrm{CHs}$ and the Base Station (BS) into the radio model. FCM-3 defines two constraints to ensures single-hop connections 
for the intra-cluster and inter-cluster communication. Similar to LEACH-3D, the number of $\mathrm{CHs}$ is variable. However, experimental results show that FCM-3 achieves the best performance when the number of CHs is from $20 \%$ to $30 \%$ of the network size, which is considered a high number.

A Particle Swarm Optimization (PSO)-based Protocol for CHs selection (PSO-CH) [5] is a centralized PSO-based protocol that is used to find the optimal set of CHs. The PSO-CH protocol considers the following properties: the network's energy efficiency, data transmission reliability, and the protocol's scalability. The objective function that is used to evaluate each candidate solution is defined as the weighted sum of three sub-objectives, each of which is related to the aforementioned properties. PSO-CH is designed and implemented under realistic networks settings and realistic energy consumption model. The link quality estimation in PSO-CH is based on the Received Signal Strength Indicator (RSSI) of received packets.

All the aforementioned protocols are applicable to 3D WSNs. However, the path loss model adapted by these protocols ignore the effect of the obstacles on the communication between the sensors. They also assume that the field is a 3D volume where the sensors can be positioned freely within the field. Moreover, all these protocols assume the use of an ideal energy consumption model.

The Surface-Level Irregular Terrain (SLIT) path loss model [4] is a semiempirical path loss model for WSN with irregular terrain. The SLIT model uses the terrain information, expressed by the DEM data, to provide a fast and an accurate estimation of the large-scale path loss due to the obstacles existing in the field. The total path loss is expressed as a function of both the free-space path loss and the path loss due to the obstacles in the field, which is calculated using the Epstein-Peterson diffraction loss model [6]. In order to verify the SLIT model, empirical experiments are conducted and the average difference between the measured results and the predicted results from the SLIT model are recorded. Experimental results have shown that the SLIT model provides an accurate path loss model that accounts for the terrain profile.

In this paper, we adopt the SLIT path loss model 4 and propose an obstacleaware clustering protocol for 3D WSNs to evaluate the effect of obstacles on clustering protocols.

\section{The System Model}

The 3D rolling field is modeled using the Digital Elevation Model (DEM). The DEM is a digital representation of a given ground surface topography or terrain. In the DEM, the network field is represented as a matrix of cells, where each cell holds a value that represents the average elevation of the area contained by that cell. DEMs are commonly built using remote sensing technology or from land surveying, and are usually available to download. For example, the geospatial data extraction tool [1] is part of Natural Resources Canada's altimetry system designed to meet the users' needs for elevation data and products. This tool provides data from seamless national datasets based on custom-defined geographic area and customized data options. The main motivation to adopt the DEM in our proposed protocol is to be able to simulate a realistic 3D rolling field and 
to find the obstacles between any two sensor nodes in the network. The sensors are assumed to be uniformly deployed in a 3D rolling field. Based on the DEM data, the height coordinate of a sensor located at position $(x, y)$ is restricted to the field's elevation at that specific position.

For the energy consumption model, a discrete-based realistic model which is based on the characteristics of the Chipcon CC2420 radio transceiver data sheet [1] is used. The total energy consumed by sensor node $n$, consumed $E_{n}$, is calculated as follows [3]:

$$
\text { consumed } E_{n}=\sum_{\text {statej }} P_{\text {statej }} \times t_{\text {statej }}+\sum_{t r} E_{\text {transitions }}
$$

The index statej refers to the energy states of the sensor: sleep, reception, or transmission. $P_{\text {statej }}$ is the power consumed in each statej, $t_{\text {statej }}$ is the time spent in the corresponding state, and $t r$ is the number of transitions for $\mathrm{S}$. The energy spent in transitions between states, $E_{\text {transitions }}$, is also added to the node's total energy consumption. The different values of $P_{\text {statej }}$ and $E_{\text {transitions }}$ can be found in [11].

For the path loss model, we adopt the SLIT path loss model [4] to find the path loss between any two sensors.

\section{An Obstacle-aware Clustering Protocol for WSNs with Irregular Terrain}

The network operating time of the proposed protocol is divided into rounds. Each round consists of two phases, the set-up phase, and the steady-state phase. The operation of these phases is similar to that of [5]. In this paper, we focus on the protocol adopted by the BS in the set-up phase to find the optimal set of $\mathrm{CHs}$ and clusters. We refer to the proposed protocol as the NSGA-based, Non-LOS $\mathrm{CH}$ selection (NSGA-NLOS-CH) protocol which includes six different processes as illustrated below:

\subsection{DEM Extraction}

The geospatial data extraction tool [1] provided by Natural Resources Canada is used to extract the elevation data for a given network field. Based on the DEM data for a given network field, the BS constructs an elevation matrix that holds the elevation data for all the cells contained by the network field. The ArcGIS software package is utilized to generate and extract the elevation data given the DEM data for that field.

\subsection{LOS Algorithm}

A Line of Sight (LOS) algorithm is needed to find the obstacles in the communication link between any two sensors in the field. In this paper, the Bresenham LOS algorithm is utilized to implement the visibility function. The Bresenham algorithm is often used in computer graphics for line drawing on $2 \mathrm{D}$ surfaces. In 
this paper, we have modified it to be used for LOS determination on 3D rolling fields. In this algorithm, if the elevation of any corresponding points between the transmitter and the receiver does not cut the virtual line drawn between them, then there is a LOS between the transmitter and the receiver. Otherwise, it is said that there is NLOS (non-LOS) between them. In this paper, an obstacle is defined as the point which has an elevation higher than that of the transmitter or the receiver. The LOS algorithm returns a visibility matrix that has $N$ rows and $N$ columns, where $N$ is the total number of sensors. This visibility matrix holds the path loss of all the obstacles between any two sensors in the network.

\subsection{Path Loss Map Calculation}

Based on the visibility matrix, a path loss map is generated by the BS. This map reflects that path loss between any two communicating sensors in the network and is calculated using the SLIT path loss model. The Castalia simulator is then modified to use this path loss map instead of the one provided by the log-normal shadow fading model to calculate the propagation loss and the RSSI values for the links between any two communicating sensors.

\subsection{Finding the Optimal Set of CHs}

Once the modified RSSI values are calculated, the BS runs an EA-based algorithm to find the optimal set of CHs. In this paper, we adopt the Non-dominated Sorting Genetic Algorithm II (NSGA-II) as an optimization tool to find the optimal set of CHs. The problem formulation for the adopted NSGA-based algorithm is provided in the next section. Table 1 presents the main notations used in this paper.

Table 1. Notations

\begin{tabular}{ll}
\hline Symbol & Definition \\
\hline$P$ & Population generated from the adopted EA \\
$N$ & Total number of sensors \\
$n$ & Sensor number $n, 0 \leq n<N$ \\
$I_{i}$ & Individual number $i$ of $P$ \\
$X_{i, n}$ & Component number $n$ of $C_{i}$ \\
$K_{i}$ & Total number of clusters generated from $C_{i}$ \\
$C L_{k_{i}}$ & Cluster number $k$ generated from $C_{i}, 0 \leq k_{i}<K_{i}$ \\
$C H_{k_{i}}$ & Cluster head number $k$ generated from $C_{i}, 0 \leq k_{i}<K_{i}$ \\
$\left|C L_{k_{i}}\right|$ & Number of sensors clustered in $C L_{k_{i}}$ \\
$E(n)$ & Remaining energy of node $n$ \\
$i n i t i a l E(n)$ & Initial energy of node $n$ \\
$R S S I_{\left(n, C H_{k_{i}}\right)}$ & RSSI value for the link from $n$ to $C H_{k_{i}}$ \\
$L Q_{\left(n, C H_{k_{i}}\right)}$ & Link quality for the link from $n$ to $C H_{k_{i}}$ \\
& $L Q_{\left(n, C H_{k_{i}}\right)}=\frac{R S I\left(n, C H_{k_{i}}\right)}{-100}$ \\
\hline
\end{tabular}


Decision Variables In the proposed protocol, a sensor node may be in one of two states: a $\mathrm{CH}$, or non-CH. To find the optimal set of $\mathrm{CHs}$, a random initial population $P$ is generated and evolved by the adopted EA. Each candidate solution (chromosome) $C_{i}$ in $P$ has a dimension equal to the network size minus the BS (i.e., $N-1$ ). Binary encoding is adopted to represent each chromosome, where the size of each component of $I_{i}$ is 1 bit.

Let, $C_{i}=\left[X_{i, 1}, X_{i, 2}, X_{i, 3}, \ldots, X_{i, N-1}\right]$ be the $i_{t h}$ chromosome of $P$ where each component, $X_{i, n}, 1 \leq n \leq N-1$ maps the state of sensor $n$. Each component $X_{i, n}$ of chromosome $C_{i}$ is initialized with either 1 to indicate that sensor $n$ is a $\mathrm{CH}$ node, or 0 to indicate that $n$ is non- $\mathrm{CH}$ node. It should be noted that this encoding process will result in a variable number of CHs. Table 2 shows the random sequences created for two individuals in $P$ on a network including 10 sensor nodes other than the BS. Each row presents a solution for a chromosome in $P$. For example, in chromosome $C_{1}$ sensors 2, 5 and 10 are CHs while the rest of the sensors are non-CH nodes.

Table 2. Chromosomes Population

\begin{tabular}{lllllllllllll}
\hline Node ID & $\mathbf{2}$ & $\mathbf{3}$ & $\mathbf{4}$ & $\mathbf{5}$ & $\mathbf{6}$ & $\mathbf{7}$ & $\mathbf{8}$ & $\mathbf{9}$ & $\mathbf{1 0}$ \\
\hline$I_{1}$ & 0 & 1 & 0 & 0 & 1 & 0 & 0 & 0 & 0 & 1 \\
$I_{2}$ & 0 & 0 & 1 & 1 & 1 & 0 & 0 & 0 & 1 & 0 \\
\hline
\end{tabular}

The clusters are formed by associating each non- $\mathrm{CH}$ node to its closest $\mathrm{CH}$ that has the lowest RSSI value. After the clustering process ends, each sensor node belongs to only one cluster and each cluster head node acts as the $\mathrm{CH}$ of exactly one cluster.

Objective Functions Each chromosome $C_{i}$ is evaluated according to four objective functions, which are briefly described in Table 3 and defined below.

Table 3. The Objective Functions

\begin{tabular}{lll}
\hline Objective Function Description & Goal \\
\hline$K_{i}$ & Minimize the total number of CHs & Save energy \\
$U_{i}$ & Minimize the number of unclustered sensors & Enhance scalability \\
$L_{i}$ & Maximize the link quality for the inter-cluster communication Data delivery reliability \\
$E_{i}$ & Maximize the total remaining energy of the CHs & Balance energy consumption \\
\hline
\end{tabular}




$$
\begin{aligned}
& K_{i}=\sum_{n=1}^{N-1} 1, \quad \text { if } X_{i, n}=1 \\
& U_{i}=N-\sum_{k_{i}=1}^{K_{i}}\left|C L_{k_{i}}\right| \\
& L_{i}=\max _{k=1,2, \ldots, K} \frac{\sum_{n \in C L_{k}} L Q_{\left(n, C H_{k_{i}}\right)}}{\left|C L_{k_{i}}\right|} \\
& E_{i}=\sum_{n=1}^{N} \frac{\text { initialE(n) }}{E(n)}, \quad \text { if } X_{i, n} \neq 00
\end{aligned}
$$

It should be noted that the calculation for $L_{i}$ in the proposed protocol depends on the newly derived RSSI values which depend on the generated path loss map.

\section{Simulation Results and Analysis}

The NSGA-NLOS-CH protocol and the proposed path loss model are implemented in Castalia. In addition, we have implemented both LEACH-3D and PSO-CH. The simulations are performed on elevation data from the Armadillo Peak volcano in British Columbia, as illustrated in Figure 1. The DEM of this field is obtained using the geospatial data extraction tool [1] provided by Natural Resources Canada. In order to generate the elevation data, a $20 \times 20$ fishnet is constructed using the ArcGIS software on a scaled version of the network field. The network field is scaled down to $100 \times 100$ meters. The sensors are deployed randomly and their position is restricted by the elevation of the rolling field. The initial energy of the sensors is set as $18720 \mathrm{~J}$. Each round is 500s and the number of rounds is 5 . We vary the total number of sensors from 100 to 500 .

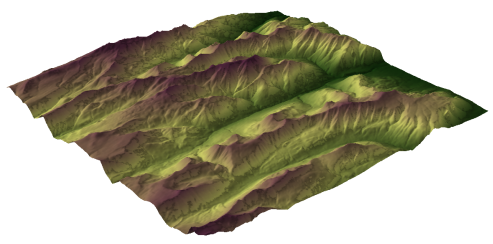

Fig. 1. Scaled version of the Armadillo Peak volcano in British Columbia

In this section, we consider the following objectives:

1. Investigate the effect of the obstacles on the Packet Delivery Rate (PDR). 
2. Compare the performance of all the competent protocols in the existence of obstacles. The comparison is done in terms of the PDR, the number of elected CHs, the energy consumption and the average number of unclustered nodes per round.

We consider two cases to investigate the effect of the obstacles on the PDR. In the first case, we assume that there is a LOS between all the sensors and that there are no obstacles in the rolling field. For this case, the log-normal shadow fading model is used to calculate the path loss and we refer to the proposed protocol as the NSGA-LOS-CH protocol. In the second case, we use the provided elevation data to find the obstacles in the field and we use the proposed obstacle-aware path loss model. For this case, we refer to the proposed protocol as the NSGA-NLOS-CH protocol. Figure 2 shows the PDR for both of these cases.

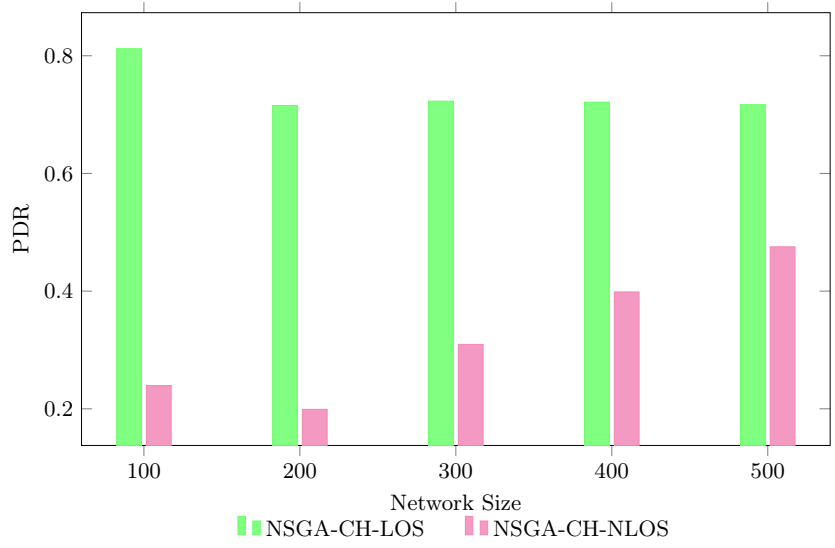

Fig. 2. PDR for NSGA-LOS-CH and NSGA-NLOS-CH

It is clearly shown that ignoring the effect of the obstacles, as in the case of NSGA-LOS-CH, can lead to more optimistic PDR values. It is also noted that the PDR of NSGA-NLOS-CH increases with the increase in the number of sensors. Increasing the number of sensors in the same network field area leads to constructing shorter links for communication with a lower probability of obstacles that could interfere those links. Next, the performance of all the competent protocols is compared in the existence of obstacles. Figure 3 shows the PDR for all the protocols. It is clearly shown that NSGA-NLOS-CH outperforms the other protocols in terms of the PDR. This is due to the fact that NSGA-NLOS$\mathrm{CH}$ clusters the network based on the RSSI values that are derived from the proposed path loss model. This leads to creating clusters that are adopted for the field profile. While PSO-CH uses the RSSI values as criteria for clustering the network, the way the RSSI calculated does not take into consideration the obstacles in the field. LEACH-3D uses a totally random mechanism when electing the CHs and this, in turn, does not guarantee a high PDR. 


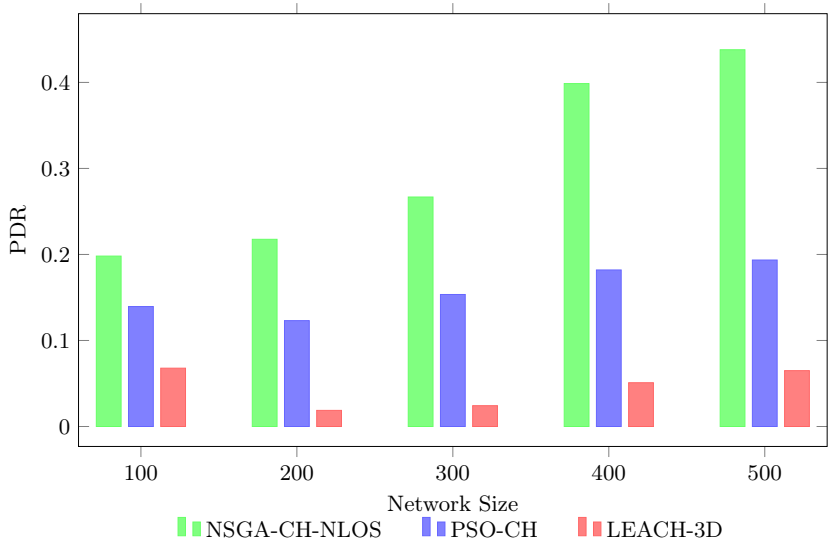

Fig. 3. PDR

Figure 4 shows the average number of CHs per round for all the protocols. It is noted that the NSGA-NLOS-CH protocol results in a higher number of CHs. Unlike PSO-CH, the number of CHs in NSGA-NLOS-CH is variable. Moreover, NSGA-NLOS-CH uses a Pareto-based approach to optimize all of its objectives concurrently. In the existence of obstacles, a higher number of $\mathrm{CHs}$ needs to cluster the whole network in order to achieve the scalability objective.

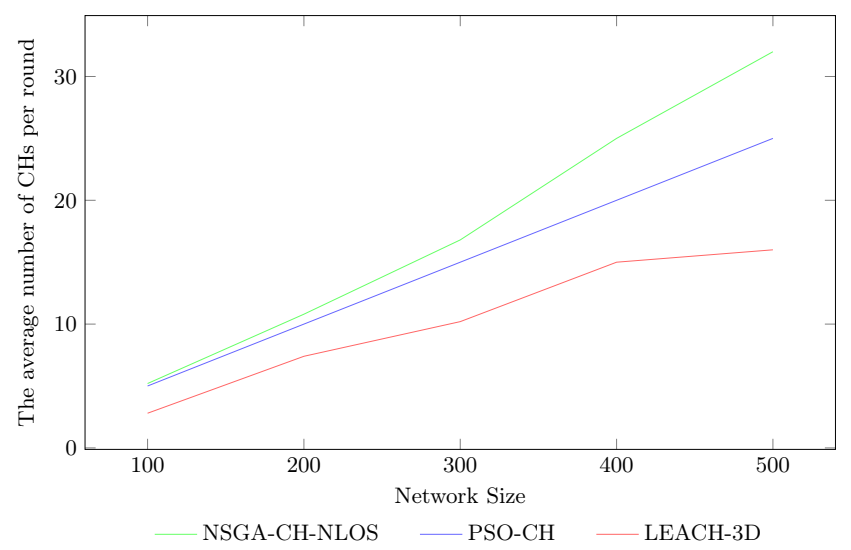

Fig. 4. The average number of $\mathrm{CHs}$ per round

The average consumed energy per sensor is shown in Figure 5. NSGA-CHNLOS has a slightly higher energy consumption than that of PSO-CH because NSGA-CH-NLOS elects a higher number of $\mathrm{CHs}$ as shown in Figure 4. These $\mathrm{CHs}$ have to stay active during the whole round which leads to a higher level of energy consumption. On the other hand, LEACH-3D has a very high energy 
consumption level. Experimental results have shown that LEACH-3D results in a very high number of unclustered sensors. These unclustered sensors stay active during the whole round and consume more energy. On the other hand, both NSGA-CH-NLOS and PSO-CH are able to cluster all the sensors. The average number of unclustered sensors per round is shown in Figure 6 .

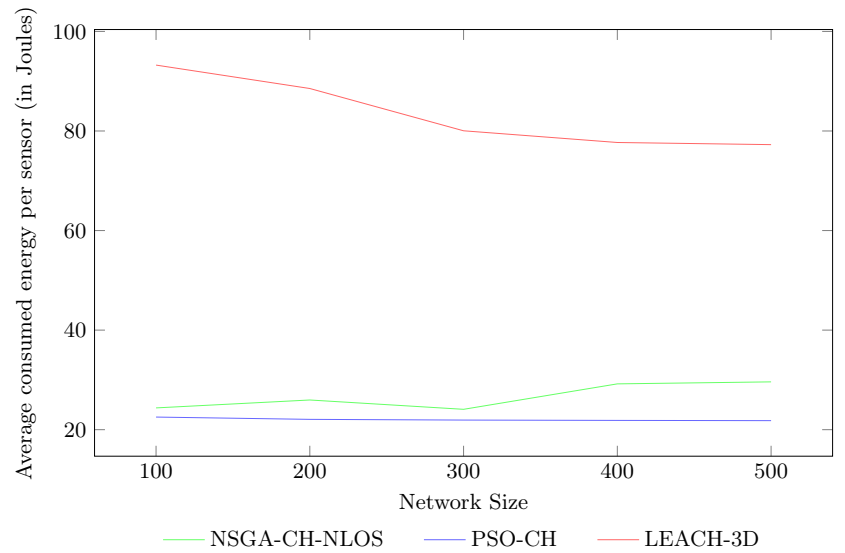

Fig. 5. The average consumed energy per sensor (in Joules)

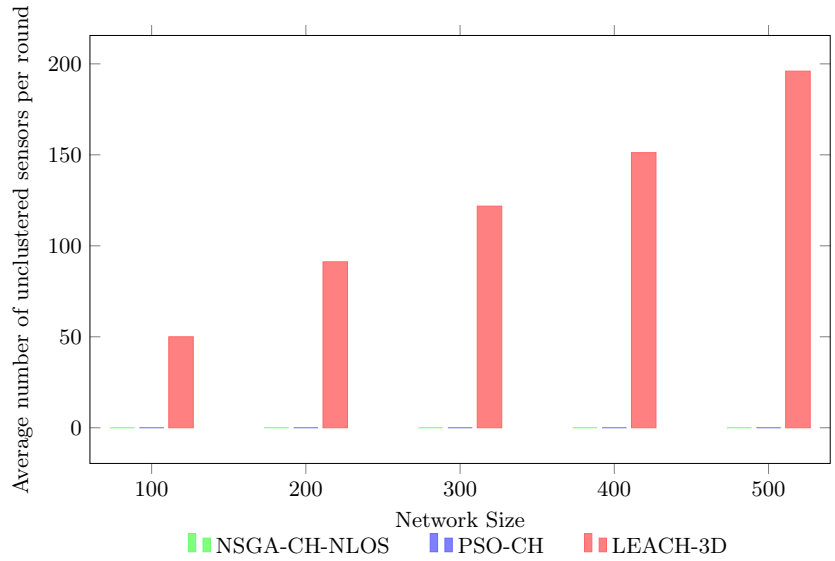

Fig. 6. The average number of unclustered sensors per round 


\section{Conclusions and Future work}

In this paper, we adopt an obstacle-aware path loss model to account for the effect of the obstacles in the network field. In order to locate those obstacles, the 3D rolling field is modeled using the DEM. Based on the adopted path loss model, an obstacle-aware clustering protocol, NSGA-NLOS-CH, is proposed. Simulation results have shown that the effect of the obstacle on the PDR cannot be neglected. Moreover, NSGA-NLOS-CH outperforms both PSO-CH and LEACH$3 \mathrm{D}$ in terms of the PDR while maintaining an acceptable energy consumption at the same time.

The coverage problem in 3D WSNs with a rolling field is another fundamental problem. As a future research direction, an integrated solution for both the clustering and coverage problems in an irregular field should be investigated.

\section{References}

1. Geospatial data extraction. http://maps.canada.ca/czs/index-en.html accessed: 2017-08-15

2. Baghouri, M., Hajraoui, A., Chakkor, S.: Low energy adaptive clustering hierarchy for three-dimensional wireless sensor network. Recent Advances in Communications pp. 214-218 (2015)

3. Barberis, A., Barboni, L., Valle, M.: Evaluating energy consumption in wireless sensor networks applications. In: 10th Euromicro Conference on Digital System Design Architectures, Methods and Tools, 2007. DSD 2007. pp. 455 -462 (Aug 2007). https://doi.org/10.1109/DSD.2007.4341509

4. Chong, P.K., Kim, D.: Surface-level path loss modeling for sensor networks in flat and irregular terrain. ACM ACM Transactions on Sensor Networks 9(2), 15:1-15:32 (Apr 2013). https://doi.org/10.1145/2422966.2422972, http://doi.acm.org/10. 1145/2422966.2422972

5. Elhabyan, R.S., Yagoub, M.C.E.: Particle swarm optimization protocol for clustering in wireless sensor networks: A realistic approach. In: Proceedings of the 2014 IEEE 15th International Conference on Information Reuse and Integration (IEEE IRI 2014). pp. 345-350 (Aug 2014). https://doi.org/10.1109/IRI.2014.7051910

6. Epstein, J., Peterson, D.W.: An experimental study of wave propagation at 850 mc. Proceedings of the IRE 41(5), 595-611 (May 1953)

7. Hai, D.T., Son, L.H., Vinh, T.L.: Novel fuzzy clustering scheme for 3d wireless sensor networks. Applied Soft Computing 54, 141 - 149 (2017). https://doi.org/https://doi.org/10.1016/j.asoc.2017.01.021

8. Kurt, S., Tavli, B.: Path-loss modeling for wireless sensor networks: A review of models and comparative evaluations. IEEE Antennas and Propagation Magazine 59(1), 18-37 (Feb 2017). https://doi.org/10.1109/MAP.2016.2630035

9. Minakov, I., Passerone, R., Rizzardi, A., Sicari, S.: A comparative study of recent wireless sensor network simulators. ACM Transactions on Sensor Networks 12(3), 20:1-20:39 (Jul 2016). https://doi.org/10.1145/2903144, http://doi.acm.org/10. $1145 / 2903144$

10. Raheemah, A., Sabri, N., Salim, M., Ehkan, P., Ahmad, R.B.: New empirical path loss model for wireless sensor networks in mango greenhouses. Computers and Electronics in Agriculture 127, 553 - 560 (2016). https://doi.org/http://dx.doi.org/10.1016/j.compag.2016.07.011

11. Texas Instruments: Chipcon CC2420 radio transceiver data sheet. http://www.ti. com/lit/ds/symlink/cc2420.pdf (2013), Last access: September 25, 2014 